\title{
The Affordable Care Act and the Burden of High Cost Sharing and Utilization Management Restrictions on Access to HIV Medications for People Living with HIV/AIDS
}

\author{
Yasamean Zamani-Hank, MPH
}

\begin{abstract}
The HIV/AIDS epidemic continues to be a critical public health issue in the United States, where an estimated 1.2 million individuals live with HIV infection. Viral suppression is one of the primary public health goals for People Living with HIV/AIDS (PLWHA). A crucial component of this goal involves adequate access to health care, specifically anti-retroviral HIV medications. The enactment of the Affordable Care Act (ACA) in 2010 raised hopes for millions of PLWHA without access to health care coverage. High cost-sharing requirements enacted by health plans place a financial burden on PLWHA who need ongoing access to these lifesaving medications. Plighted with poverty, Detroit, Michigan, is a center of attention for examining the financial burden of HIV medications on PLWHA under the new health plans. From November 2014 to January 2015, monthly out-of-pocket costs and medication utilization requirements for $31 \mathrm{HIV}$ medications were examined for the top 12 insurance carriers offering Qualified Health Plans on Michigan's Health Insurance Marketplace Exchange. The percentage of medications requiring quantity limits and prior authorization were calculated. The average monthly out-of-pocket cost per person ranged from $\$ 12$ to $\$ 614$ per medication. Three insurance carriers placed all 31 HIV medications on the highest cost-sharing tier, charging $50 \%$ coinsurance. High out-ofpocket costs and medication utilization restrictions discourage PLWHA from enrolling in health plans and threaten interrupted medication adherence, drug resistance, and increased risk of viral transmission. Health plans inflicting high costs and medication restrictions violate provisions of the ACA and undermine health care quality for PLWHA. (Population Health Management 2016;19:272-278)
\end{abstract}

\section{Introduction}

$\mathbf{T}$ HE HIV/AIDS EPIDEMIC CONTINUES to be a critical public health issue in the United States. It is estimated that nearly 1.2 million individuals in the United States were infected with HIV in 2011. ${ }^{1}$ Of this total, $86 \%$ were diagnosed with the infection, yet only $40 \%$ were engaged in medical care, and only $30 \%$ were virally suppressed. ${ }^{1}$ The other $60 \%$ diagnosed with HIV who are not engaged in care are at an increased risk of experiencing complications from uncontrolled viral loads, such as increased vulnerability to bacterial infections, as well as cardiovascular, liver, neuropathic, and kidney problems. ${ }^{2}$ Individuals who cannot suppress their viral loads are also at increased risk of acquiring AIDS and transmitting the virus to others. ${ }^{2,3,4}$ Viral load suppression is one of the primary public health goals for People Living with HIV/AIDS (PLWHA), and a high priority for maintaining and ensuring a high quality of life and wellbeing for these individuals. ${ }^{5,6}$ The US Centers for Disease Control and Prevention (CDC) estimates that $76 \%$ of individuals who are in care achieve viral load suppression, highlighting the importance of encouraging HIV-infected individuals to get into care as soon as possible, and stay in care. ${ }^{5}$

The importance of getting into care and staying in care underscores the necessity of adequate health care coverage for PLWHA. ${ }^{1,7}$ The enactment of the Patient Protection and Affordable Care Act (PPACA), also known as the Affordable Care Act (ACA), in 2010 has promoted access to health care coverage for millions of Americans who had no previous source of health care. ${ }^{7}$ The 2 most significant benefits for individuals living with HIV/AIDS have been the opportunity to purchase private health insurance through state marketplace insurance exchanges, and the ability to enroll

Department of Health Behavior \& Health Education, University of Michigan School of Public Health, Ann Arbor, Michigan. 
without fear of denial of coverage, or annual limits on benefits, related to a preexisting health condition or their current health status. ${ }^{7}$ The health insurance exchanges offer a variety of bronze, silver, and gold plans that represent different cost-sharing requirements but must offer the same core essential health benefits required by the ACA. ${ }^{8}$ The availability of new private health plans and the elimination of discriminatory insurance practices represent 2 major hallmarks of the ACA with tremendous social, political, and historical importance for PLWHA.

Despite the prohibition of discriminatory insurance practices under the ACA, recent concerns have been raised regarding the structuring of health care benefits by certain insurance companies that impose excessive financial and access restrictions to important health care services, including medication acquisition. ${ }^{9}$ In May 2014, the AIDS Institute and the National Health Law Program filed an official complaint with the Office for Civil Rights in the United States Department of Health and Human Services against 4 Florida insurers for engaging in insurance practices that actively discouraged PLWHA from enrolling in their plans. ${ }^{9}$ CoventryOne, Cigna, Humana, and Preferred Medical structured their drug formularies in a way such that all HIV/AIDS medications were placed on the highest cost-sharing tier, and also enacted medication restrictions such as prior authorization and quantity limits. ${ }^{9}$ These restrictions can serve as barriers to adequate access to anti-retroviral therapy (ART) medications for PLWHA. ${ }^{9,10}$

Excessive high costs for HIV medications place a financial burden on PLWHA who need ongoing access to these life-saving medications, and can discourage them from enrolling in health care plans. ${ }^{9}$ As it is estimated that $87 \%$ of individuals in care in the United States with HIV between the ages of 19-64 have incomes below $400 \%$ of the federal poverty level, the issue of financial access to medications is crucial to address. ${ }^{7}$ The relationship between socioeconomic status (SES) and HIV is of critical public health concern, as people with lower SES are disproportionately impacted by HIV infection. ${ }^{11,12}$ Poverty and low SES are risk factors for HIV infection, as people with inadequate access to socioeconomic resources may engage in riskier sexual practices that can lead to HIV contraction. ${ }^{11,12,13}$ In particular, lack of access to medical care heavily influences access to needed medications and appropriate medication adherence. ${ }^{14}$ Poor medication adherence can lead to reduced health outcomes for PLWHA and an increase in drug resistant HIV strains that can be transmitted to others. ${ }^{3,15,16}$

Because of the strong connection between poverty and vulnerability to HIV infection, prodigious attention has been focused on the HIV/AIDS epidemic in the impoverished city of Detroit, Michigan, a region devastated by economic and financial struggle in recent years. According to 2013 estimates, Detroit has the highest HIV prevalence rate in Michigan, at 7040 per 100,000 individuals. ${ }^{17}$ Furthermore, $64 \%$ of individuals infected with HIV in Michigan reside in Detroit at the time of diagnosis. ${ }^{17}$ There is also a disproportionate impact of HIV on African American men in Detroit, particularly among African American men who have sex with men. ${ }^{17}$ This population has the highest HIV prevalence rate in the state, and also the highest number of PLWHA. ${ }^{6}$ Because of the high prevalence rate of HIV/ AIDS among underserved and vulnerable populations in
Detroit, the issue of financial access to comprehensive HIV health care needs is a crucial one that needs to be addressed.

The Ryan White HIV/AIDS Program (RWHAP) has been essential to filling the gaps in health care coverage for PLWHA. The Ryan White legislation was passed by Congress in 1990 to promote health care services for PLWHA who have insufficient financial resources to obtain needed medical care and support services for their HIV condition. ${ }^{18}$ The legislation was named in honor of a young boy named Ryan White who was diagnosed with AIDS at the age of 13 after he underwent a blood transfusion procedure needed for his hemophiliac condition. The RWHAP continues to serve as a critical safety net for PLWHA who do not qualify for expanded Medicaid programs or the federally facilitated health insurance marketplace exchange (ACA marketplace exchange) ${ }^{7,18}$ An individual living with HIV/AIDS may be eligible for expanded Medicaid, also known as the Healthy Michigan Plan, if he or she is at or below $133 \%$ of the federal poverty level. ${ }^{19}$ However, if an individual living with HIV/AIDS is not within these income requirements, he or she would not be eligible for Medicaid and may proceed to look for coverage through the marketplace exchange, which does not have an income requirement. In order to be eligible for the marketplace exchange, one must be a US citizen living in the United States, not currently incarcerated, and not have access to health insurance coverage elsewhere ${ }^{20}$ There are no income requirements in order to be eligible for the marketplace, although financial assistance is available for individuals who qualify. However, it is important to recognize that even among PLWHA who do qualify for the health insurance marketplace exchange, the Ryan White Programs play a crucial role in assisting with the payment of prescription drug co-payments as well as insurance plan premiums and deductibles charged by the Qualified Health Plans (QHPs).

The lines delineating who is eligible for the marketplace exchange and who is eligible for the Ryan White programs are not always clear cut. In other words, it is possible for PLWHA to obtain both exchange coverage and Ryan White coverage. The ACA marketplace exchange offers financial assistance in the form of premium tax credits for individuals within $100 \%-400 \%$ of the federal poverty level to help lower monthly premium payments, and also cost-sharing subsidies to reduce co-payments for doctor's visits and prescription drugs for individuals who are within 100\%-250\% of the federal poverty level. ${ }^{7}$ However, despite these financial assistance programs offered by the ACA marketplace, prescription drug co-payments, premiums, and deductibles still may be a formidable barrier for low-income PLWHA. For a 55-year old individual living with HIV/AIDS with an annual income of $\$ 17,655$ (150\% of the federal poverty level) in Detroit, Michigan, the monthly premium for a health plan on the exchange may cost up to $\$ 345$ per month. This is where the Ryan White Program steps in to assist individuals with these costs. The Ryan White Program consists of 5 different programs (Parts A-F), which provide funding to multiple types of entities such as state and local health departments, research institutions, as well as community-based organizations. ${ }^{18}$ This funding is used to provide diverse health care services to various populations of individuals living with HIV (eg, all adults, pregnant women, infants, children, metropolitan area residents). ${ }^{18}$ Each program within Ryan White 
may target a different population of PLWHA, and the income eligibility requirements of the Ryan White Programs may vary by state and program. In Michigan, with some exceptions, the Ryan White Programs will cover individuals living with HIV/AIDS who are at or below $450 \%$ of the federal poverty level.

The Part B Program of the Ryan White Act is critical to this discussion of affordable prescription HIV drug coverage for PLWHA. This program provides funding for the State AIDS Drug Assistance Programs for PLWHA who need financial assistance paying for their HIV medication copayments, insurance plan premiums, or insurance plan deductibles. In order to be eligible for the Ryan White-funded Michigan Drug Assistance Program (MIDAP), a person must provide proof of HIV status, must be a resident of Michigan, and must be at or below $450 \%$ of the federal poverty level. ${ }^{21}$ In some cases, eligible individuals also must show that they have applied for public assistance programs such as Medicaid and have either a pending or denial status. This criterion is to ensure that Ryan White Funds are utilized in a cost-effective way by targeting PLWHA who truly have no other source of coverage to help cover the costs of their medications. MIDAP may help pay for the premiums, deductibles, and co-payments of medications charged by Qualified Health Plans (QHPs) on the marketplace exchange. However, for low-income individuals living with HIV/AIDS who may not qualify for the marketplace exchange (ie, undocumented citizens, selfemployed, individuals who do not qualify for Medicaid), or any other type of health insurance, MIDAP can provide "full coverage" to these individuals and pay for the full cost of their HIV medications in the absence of insurance coverage. Moreover, other individuals who may already have health insurance through the marketplace may qualify under MIDAP to receive co-payment, premium, and deductible assistance. Undoubtedly, there is a great deal of eligibility overlap between the exchanges and the Ryan White programs, which ensures that low-income PLWHA are not falling through the cracks and becoming lost to care.

In Michigan, of the estimated 8809 individuals with HIV/ AIDS who are enrolled in the RWHAP, $75.9 \%$ have incomes below $100 \%$ of the federal poverty level. ${ }^{22}$ These statistics point to the fact that financial access to HIV care services poses a barrier for PLWHA. Financial barriers to essential HIV medications in Michigan continue to be a critical issue. Access to life-saving ART medications is one of the most crucial components of appropriate health care services for PLWHA. ${ }^{1,3}$ Adequate access to ART for PLWHA is a top priority for Michigan's Statewide Coordinated Statement of Need and CDC's strategic HIV prevention plan. ${ }^{6,17}$ The prominent need for HIV Care services in Michigan generally, and Detroit in particular, underscores the importance of examining the impact of the ACA in making health care more affordable for this population of great need in Michigan. Because of the dearth of studies examining the coverage of HIV/AIDS medications on the drug formularies of QHPs on the Michigan Health Insurance Marketplace Exchange, this article presents a first of its kind cost comparison of the out-of-pocket costs of HIV medications charged by 12 major insurance carriers in Detroit, Michigan.

\section{Methods}

Between November 2014 and January 2015, the drug formularies of 12 major insurance carriers offering silver QHPs in Wayne County on the Michigan Health Insurance Marketplace Exchange were analyzed. The following 12 carriers are the major health insurance companies offering plans on the marketplace in the entire State of Michigan: Priority Health, Blue Cross Blue Shield, Blue Care Network, Health Alliance Plan (HAP), McLaren Health Plan, Total Health Care USA Inc, Consumer's Mutual of Michigan, Assurant Health, United Healthcare, Humana, Harbor Health Plan, and Molina Healthcare of Michigan. Forty-nine silver QHPs are offered in Wayne County on the 2015 Michigan Marketplace Exchange. The drug formularies were located on the insurance companies' Web sites. Using the drug formulary criteria of the MIDAP, the author calculated the monthly out-of-pocket cost per person charged by the insurance carriers for each of the 31 ART HIV medications examined. At the time this research was performed, the MIDAP formulary covered 31 ART medications.

The 31 drugs examined were Abacavir (Ziagen), Abacavir/ lamivudine (Epzicom), Abacavir/lamivudine/zidovudine (Trizivir), Atazanavir (Reyataz), Darunavir (Prezista), Delavirdine (Rescriptor), Didanosine (Videx), Dolutegravir (Tivicay), Efavirenz (Sustiva), Efavirenz/emtricitabine/tenofovir (Atripla), Elvitegravir/cobicistat/emtricitabine/tenofovir (Stribild), Emtricitabine(Emtriva), Emtricitabine/tenofovir (Truvada), Emtricitabine/rilpivirine/tenofovir (Complera), Enfuvirtide (Fuzeon), Etravirine (Intelence), Fosamprenavir (Lexiva), Indinavir (Crixivan), Lamivudine (Epivir), Lamivudine/ zidovudine (Combivir), Maraviroc (Selzentry), Nelfinavir (Viracept), Nevirapine (Viramune), Raltegravir (Isentress), Rilpiviring (Edurant), Ritonavir (Norvir), Saquinavir (Invirase), Stavudine (Zerit), Tenofovir (Viread), Tipranavir (Aptivus), and Zidovudine (Retrovir). ${ }^{23}$ The HIV medications are identified by their generic name, with corresponding brand names in parentheses. The drug formularies categorized medications according to tier level, which determines whether a drug is a generic medication, a preferred brand medication, a non-preferred brand medication, a preferred specialty medication, or a non-preferred specialty medication. The tier categorization dictates the cost-sharing obligations for the medications. Although tier levels vary slightly between insurance carriers, levels are usually categorized on 4 or 5 tier levels. A higher tier level generally corresponds to a higher co-payment (co-pay) or coinsurance cost for the medication. A co-pay is a fixed dollar amount that a patient is obliged to pay each time a medical service is obtained, whereas a coinsurance is a fixed percentage of the total medical service bill that a patient is obliged to pay each time a medical service is obtained. Generally speaking, a copay is less expensive than coinsurance, because coinsurance will increase as the cost of the medical service increases, whereas a co-pay will always remain the same. Tier levels 3 and above were considered to be higher tier levels. Specialty Drug Tier Levels (usually 4 or 5) were considered to be the highest tier level.

The estimated retail cost of the drug was derived from the PositivelyAware (PA) HIV Drug Guide. ${ }^{24}$ PA is a notfor-profit HIV/AIDS treatment journal published by Test Positive Aware Network, a not-for-profit AIDS Service 
Organization based in Chicago. The PA site has been used as a trusted resource by various federally funded State Drug Assistance Programs, such as Michigan and Illinois, as well as nonprofit organizations such as the AIDS Foundation of Chicago, to estimate monthly drug costs for clients on the drug assistance programs and to assist with cost-effective analyses in state program planning. Because insurance plans offered on the marketplace are not always transparent about the actual out-of-pocket costs for HIV medications, the PA site acts as a trusted substitute in calculating estimated outof-pocket costs for expensive HIV medications. Hence, the PA site was used in this research as the select resource for estimating HIV drug costs.

Based on the PA drug guide's estimated monthly retail costs for the medications, the co-pay or coinsurance cost charged by the health insurance plan was applied in order to determine the estimated out-of-pocket cost per person per month for the HIV medications examined. The co-pay or coinsurance cost for each silver plan was obtained from the Michigan Marketplace healthcare.gov. The average out-ofpocket cost per person per month was calculated for all HIV medications covered by each insurance carrier. The drug formularies of each insurance carrier were analyzed to determine the total number of covered HIV medications and the number of HIV medications with utilization management restrictions such as quantity limits and prior authorizations. Numbers are displayed as percentages in Figures 1-5.

\section{Results}

Results found that the overall coverage of HIV medications is extremely high among the 12 insurance carriers, ranging from $90.3 \%$ coverage to $100 \%$ coverage. Humana, Harbor Health Plan, United Healthcare, Consumer's Mutual, Blue Cross Blue Shield, Blue Care Network, McLaren Health Plan, and Assurant Health covered $100 \%$ of HIV medications. Priority Health and Molina covered $96.8 \%$ and HAP covered $90.3 \%$. Because these are among the most prominent insurance carriers in Michigan that offer Qualified Health Plans on the marketplace exchange, it is reassuring to know that HIV medication coverage on drug formularies is high.

Figure 1 illustrates the average out-of-pocket cost per person per month charged by each insurance carrier for the

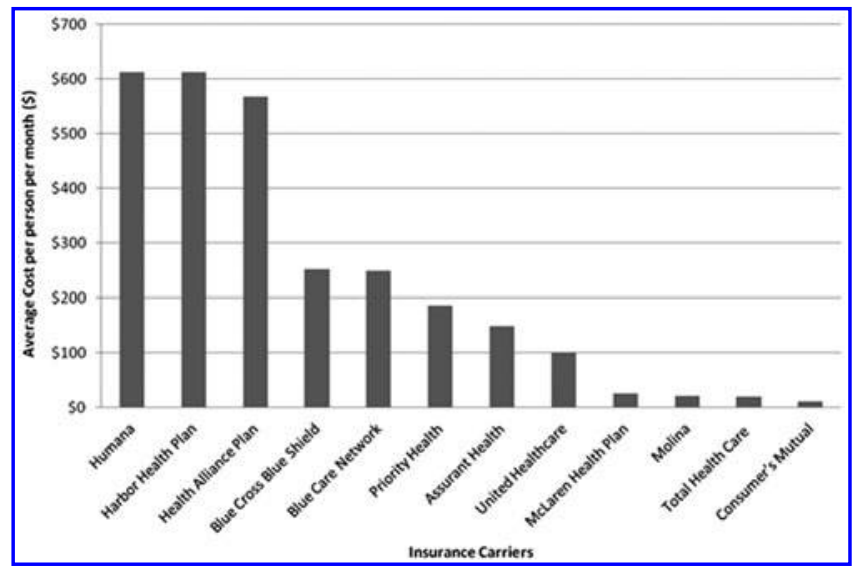

FIG. 1. The average out-of-pocket cost per person charged by insurance carriers for HIV medications.

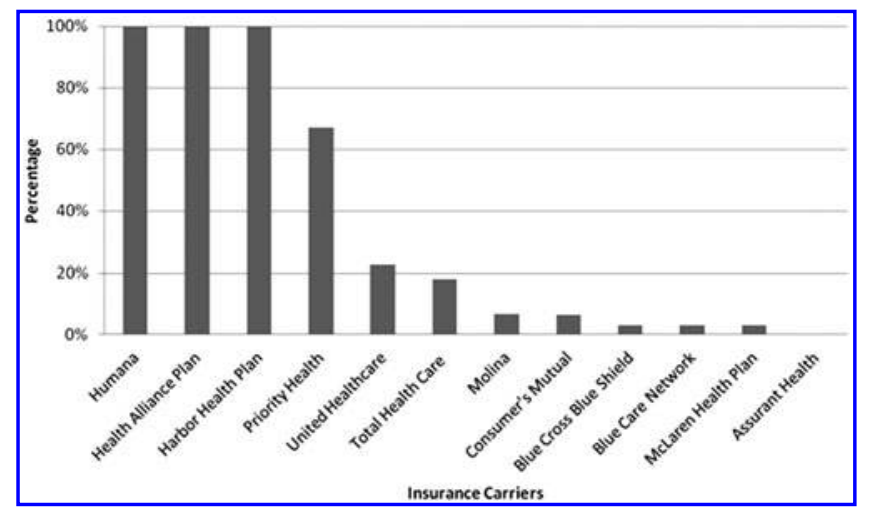

FIG. 2. The percentage of HIV drugs placed on higher tier levels on the drug formulary of each insurance carrier.

31 HIV medications examined. As illustrated by Figure 1, Humana, Harbor Health Plan, and HAP charge the highest out-of-pocket costs, ranging from $\$ 567$ to $\$ 614$ per person per month per HIV medication. Blue Cross Blue Shield, Blue Care Network, Priority Health, Assurant Health, and United Healthcare charge lower average out-of-pocket costs per person per month, ranging from \$101 to \$252. McLaren Health Plan, Molina, Total Health Care, and Consumer's Mutual charge the lowest average out-of-pocket costs per person per month. These results indicate the large discrepancy in co-pay and coinsurance costs charged by insurance carriers for the same HIV medications.

Figure 2 illustrates the percentage of HIV medications placed on higher cost-sharing tier levels. Eleven of the 12 insurance carriers placed more than $1 \mathrm{HIV}$ medication on the higher cost-sharing tiers. Three of the 12 insurance carriers placed all $31 \mathrm{HIV}$ medications on higher tier levels. Three of the 12 insurance carriers, Humana, HAP, and Harbor Health Plan, placed every single HIV medication on the highest (specialty) tier level, meaning that clients would be paying the highest out-of-pocket cost for these medications. These 3 insurance carriers charge $50 \%$ coinsurance for these HIV specialty drugs, the highest coinsurance charged by any of the insurance carriers examined.

Figure 3 depicts the percentage of HIV drugs on the drug formularies that require quantity limits by each insurance carrier. Humana, Assurant Health, and Total Health Care required quantity limits for all $31 \mathrm{HIV}$ medications examined.

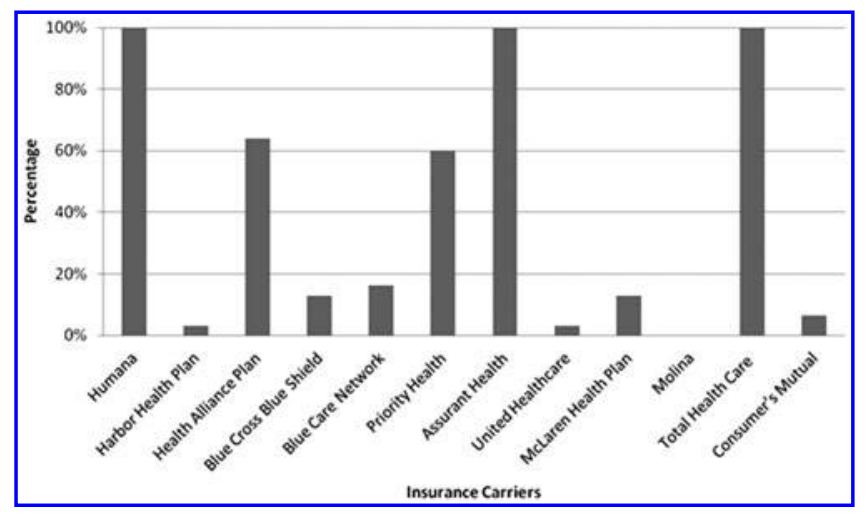

FIG. 3. The percentage of HIV drugs with quantity limits on the drug formulary of each insurance carrier. 


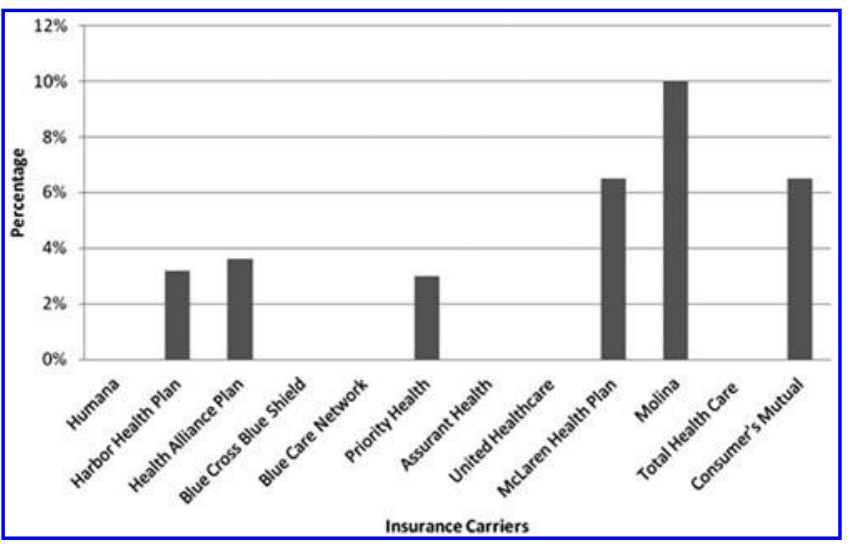

FIG. 4. The percentage of HIV medications that require prior authorization by insurance carrier.

Eleven of the 12 insurance carriers required quantity limits for more than 1 covered HIV medication. Five of the 12 insurance carriers required quantity limits for more than half of covered HIV medications on their formularies.

Figure 4 shows the percentage of HIV medications requiring prior authorizations by the insurance carriers. Six of the 12 insurance carriers require prior authorizations for some of the HIV medications covered on their formularies. Figure 5 shows the coinsurance charged by each insurance carrier for specialty HIV drugs. Three insurance carriers (Humana, HAP, Harbor Health Plan) all charged 50\% coinsurance for HIV drugs categorized under the specialty tier. Total Health Care, McLaren, and Assurant Health were excluded as they did not charge coinsurance for their specialty medications.

\section{Discussion}

Two of the main tenets of the ACA with tremendous significance for PLWHA are improved access to affordable health care coverage and the elimination of discriminatory insurance practices toward people with preexisting conditions and other significant health issues. ${ }^{7}$ These results show that although overall coverage of HIV medications is high on the drug formularies examined, the high out-of-pocket costs charged by some insurance carriers may still pose a

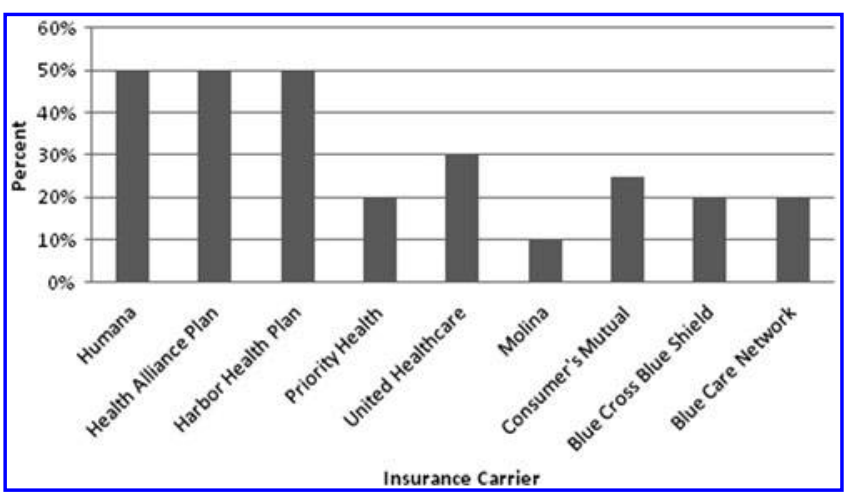

FIG. 5. The coinsurance charged for specialty drug tier. Total Health Care, McLaren, and Assurant Health were excluded as they did not charge coinsurance for their specialty medications. serious financial barrier to socioeconomically deprived PLWHA living in the Detroit Metropolitan Area. As $75.9 \%$ of PLWHA in RWHAP care in Michigan have household incomes below $100 \%$ of the federal poverty level, the high out-of-pocket costs for HIV medication acquisition undoubtedly serve as a barrier to adequate health care access. ${ }^{22}$ To help buffer the costs of health care coverage and increase incentives for enrollment of financially deprived individuals, the ACA offers premium tax credits for individuals with incomes within $100 \%-400 \%$ of the federal poverty level as well as cost-sharing subsidies to individuals with incomes within $100 \%-250 \%$ of the federal poverty level who enroll in silver plans. ${ }^{7}$

Despite these subsidies, the results of this study demonstrate that the out-of-pocket costs for HIV medications may still pose a serious financial barrier for socioeconomically deprived PLWHA. As PLWHA are often required to take multiple HIV medications or combined pills as part of their treatment regimen, they may be spending more than half of their monthly income on just HIV medications. According to Jonathan Cohn, MD, MS (oral communication, August 2015), Director at Wayne State University's Adult HIV/ AIDS program located in Detroit, Michigan, the state's largest institution providing health care services to PLWHA, the HIV drugs Stribild and Complera are some of the most common single tablet regiments taken by PLWHA. These 2 HIV drugs are categorized as combination Nucleoside/ Nucleotide Reverse Transcriptase Inhibitors (NNRTIs) and Non-Nucleoside Reverse Transcriptase Inhibitors (NRTIs), also referred to as NNRTI/NRTI combination. Combined, single-tablet pill regimens have been adopted as the preferred form of HIV medication intake by many PLWHA and infectious disease providers, as they have been studied to reduce pill burden and encourage medication adherence. ${ }^{25}$ According to PA, the average cost for Stribild and Complera per month is $\$ 2463.37$ each. For an individual living with HIV/AIDS under a Humana insurance plan, which charges a coinsurance of $50 \%$ as found by the current research, the total out-of-pocket cost for an individual on Stribild or Complera would be about $\$ 1231.69$ per month. For an individual with an income of $150 \%$ of the FPL, making about $\$ 1471.25$ a month, an average monthly cost of $\$ 1231.69$ for 1 HIV medication would represent about $84 \%$ of their monthly income. That is just the beginning of their expenditures. In addition to covering the costs of their necessary life-saving medications, PLWHA also must cover the costs of needed laboratory tests, medical appointment visits, case management services, dental care needs, and everyday expenses such as transportation, housing, and nutrition. ${ }^{26}$ For an individual living with HIV/AIDS with an income at or below $150 \%$ of the federal poverty level, the monthly costs of their medications are grossly disproportionate in the scope of other needed living expenses.

Despite the promise of expanded insurance coverage under the ACA, the cost-sharing fees for these HIV medications are undoubtedly prohibitive under many health plans offered on the marketplace exchange. Higher cost sharing for HIV medications may inhibit PLWHA from obtaining their medications in a timely manner. The implications of this may be profound, as interrupted medication adherence may increase the risk of developing drug resistance, transmitting the virus to others, and even death. ${ }^{1,3,10,27,28}$ The 
results of the current study demonstrate that many of the insurance carriers imposed utilization management restrictions for covered HIV medications. Utilization management restrictions such as prior authorization may reduce accessibility to treatment by increasing the likelihood that patients experience a delay in receipt of their medications, leading to an interruption in their medication regimen. ${ }^{10}$ Continuous access and adherence to HIV medications are crucial components for achieving the goal of viral load suppression and improving health outcomes among PLWHA. ${ }^{1}$ Even short periods of interrupted or inadequate care may drastically increase mortality risk among PLWHA. ${ }^{27}$

Targeting PLWHA with excessive utilization management requirements and exorbitant out-of-pocket costs for needed HIV medications also has the effect of discouraging PLWHA from enrolling in health insurance, which represents a violation of the ACA. ${ }^{9} 42$ USC Section 18031(c)(1)(a) states that health plans offered on the marketplace exchange cannot “...employ marketing practices or benefit designs that have the effect of discouraging the enrollment in such plan by individuals with significant health needs. ${ }^{, 9,29}$ Categorizing HIV medications on higher tier levels that require higher cost sharing and imposing prior authorization and quantity limits certainly constitute discouragement of PLWHA from enrolling in QHPs. ${ }^{9}$

These issues all point to the potential unanticipated consequences of the ACA for PLWHA. In addition to the disruptions in medication adherence and access posed by high out-of-pocket costs and utilization management requirements of QHPs, difficulties in accessing and understanding drug formularies and cost-sharing obligations highlight the need to hold health insurance companies accountable for transparency of their drug formularies.

It is important to comment on the difficulty of locating the correct drug formularies. As this study found, links to drug formularies on the Michigan marketplace exchange often led to the home page of the insurance company instead of directly to the formulary. Therefore, considerable extra time and effort were required in order to search for the drug formulary from that site. On average, the drug formulary documents were 178 pages in length. Therefore, considerable time and effort to review and locate the necessary HIV medications was required. Moreover, locating the drug formularies required access to a computer and adequate Internet technology skills, which may be a barrier for socioeconomically deprived PLWHA in cities such as Detroit who may not have the resources to access a computer or the literacy skills to analyze a drug formulary. Low SES is a risk factor for poor health literacy skills. ${ }^{30}$ It is estimated that $47 \%$ of adults in Detroit are considered functionally illiterate. ${ }^{31-33}$ This means that these adults have poor reading, speaking, and writing skills. Individuals with low health literacy skills are more likely to have poor adherence to their medication regimen, poor comprehension of drug warning labels, and decreased involvement in health promoting behaviors. ${ }^{34,35}$ Kalichman et al (2005) found poorer medication adherence and more adverse health outcomes among people living with HIV/AIDS who had lower health literacy skills. ${ }^{36}$ Undoubtedly, the tedious format in which some drug formularies are provided may serve as a large barrier to care and medication adherence for individuals of lower health literacy residing in Detroit. Future efforts need to be made on the part of insurance carriers to break down information regarding drug coverage and drug costs in a manner that can better facilitate informed decision making and insurance plan selection among individuals with diverse health literacy skills.

Although the corresponding tier levels were displayed for each medication on the drug formularies, the monthly outof-pocket costs were not made available. This is a significant shortcoming in the current approach, as it prevents PLWHA from gaining an understanding of what their estimated cost-sharing obligation will be. These asymmetries show that adequate health literacy skills and access to a computer are needed for enhanced understanding of health benefits and services offered by QHPs on the marketplace exchange. With a majority of HIV/AIDS cases disproportionately distributed in the Detroit Metropolitan Area, ensuring that vulnerable PLWHA not only have financial access to life-saving medications, but also clear and comprehensive information about what health care benefits and services are included in their QHPs, is critical to maintaining and enhancing quality of life for PLWHA in Michigan.

These findings suggest that the assurance of providing PLWHA with adequate and clear information about the covered health benefits and cost-sharing obligations for medications is crucial to informed decision making and selection of a plan that best meets one's health care needs. These findings also suggest that continuous analysis and monitoring of drug formularies is necessary to ensure that insurance company practices are consistent with the legal requirements of the ACA and to hold them accountable for insurance benefit designs that take advantage of vulnerable populations like PLWHA.

\section{Author Disclosure Statement}

Ms. Zamani declared no conflicts of interest with respect to the research, authorship, and/or publication of this article. This research received no specific grant from any funding agency in the public, commercial, or not-for-profit sectors.

\section{References}

1. Bradley H, Hall HI, Wolitski RJ, Van Handel MM, Stone AE, et al. Vital Signs: HIV diagnosis, care, and treatment among persons living with HIV-United States, 2011. Centers for Disease Control and Prevention: Morbidity and Mortality Weekly Report. 2014;63(47):1113-1117. Available at: http://www.cdc.gov/mmwr/preview/mmwrhtml/ mm6347a5.htm. Accessed February 26, 2014.

2. Ferry T, Raffi F, Collin-Filleul F, et al. Uncontrolled Viral Replication as a Risk Factor for Non-AIDS Severe Clinical Events in HIV-Infected Patients on Long-Term Antiretroviral Therapy: APROCO/COPILOTE (ANRS CO8) Cohort Study. J Acquir Immune Defic Syndr. 2009:51:407-415.

3. McManus KA, Engelhard CL, Dillingham R. Current challenges to the United States' AIDS drug assistance program and possible implications of the affordable care act. http://www.hindawi.com/journals/art/2013/350169/. Accessed August 27, 2015.

4. Reekie J, Gatell JM, Yust I, et al. Fatal and non-fatal AIDS and non-AIDS events in HIV-1 positive individuals with high CD4 counts according to viral load strata. AIDS. 2011; 25:2259-2268.

5. Centers for Disease Control and Prevention. CDC Vital Signs: HIV Care Saves Lives. 2014. Available at: http:// 
www.cdc.gov/VitalSigns/hiv-aids-medical-care/index.html. Accessed February 26, 2015.

6. Michigan Department of Community Health; Division of Health Wellness and Disease Control. Michigan's Statewide Coordinated Statement of Need and HIV/AIDS Comprehensive Plan 2012-2015. https://www.michigan .gov/documents/mdch/2012_SCSN_Comprehensive_Plan_ 399346_7.pdf. Accessed January 6, 2015.

7. Kates J, Garfield R, Young K, Quinn K, Frazier E, Skarbinski J. Assessing the impact of the affordable care act on health insurance coverage of people with HIV. 2014. https://kaiserfamilyfoundation.files.wordpress.com/2013/12/ 8535-assessing-the-impact-of-the-affordable-care-act-onhealth-insurance-coverage.pdf. Accessed August 12, 2014.

8. Healthcare.gov. 10 health care benefits covered in the health insurance marketplace. 2013. https://www.healthcare .gov/blog/10-health-care-benefits-covered-in-the-healthinsurance-marketplace/. Acessed March 1, 2015.

9. National Health Law Program. NHeLP and The AIDS Institute Complaint to HHS Re HIV/AIDS Discrimination by Florida Insurers. 2014. http://www.healthlaw.org/ publications/browse-all-publications/HHS-HIV-Complaint\# .VRoBAfnF-8B. Accessed January 20, 2015.

10. Raper JL, Willig JH, Lin H, et al. Uncompensated medical provider costs associated with prior authorization for prescription medications in an HIV clinic. Clin Infect Dis. 2010;51:718-724.

11. American Psychological Association. HIV/AIDS and Socioeconomic Status. 2015. http://www.apa.org/pi/ses/ resources/publications/factsheet-hiv-aids.aspx. Accessed March 29, 2015.

12. Perry MJ. Gender, race and economic perspectives on the social epidemiology of HIV infection: implications for prevention. Journal of Primary Prevention. 1998;19(2):97-104.

13. Santelli JS, Lowry R, Brener ND, Robin L. The association of sexual behaviors with socioeconomic status, family structure, and race/ethnicity among US adolescents. Am J Public Health. 2000;90:1582-1588.

14. Hoang T, Goetz MB, Yano EM, et al. The impact of integrated HIV care on patient health outcomes. Med Care 2009;47:560-567.

15. Hecht F, Grant R, Petropoulos C, et al. Sexual transmission of an HIV-1 variant resistant to multiple reversetranscriptase and protease inhibitors. N Engl J Med. 1998; 339(5):307-311.

16. Veenstra J, Schuurman R, Cornelissen M, et al. Transmission of zidovudine-resistant HIV-1 following deliberate injection of blood from a patient with AIDS: characteristics and natural history of the virus. Clin Infect Dis. 1995;21: $556-560$

17. Michigan Department of Community Health. Annual HIV Surveillance Report, Michigan. 2014. http://www.michigan .gov/documents/mdch/July_2014_full_report_465192_7.pdf. Accessed February 20, 2015.

18. Health Resources and Services Administration. About the Ryan White HIV/AIDS Program. 2011. http://hab.hrsa.gov/ abouthab/aboutprogram.html. Accessed March 1, 2015.

19. Michigan Department of Health and Human Services. Healthy Michigan Plan: Who is Eligible. 2015. http:// www.michigan.gov/healthymiplan/0,5668,7-326-67874-,00 .html. Accessed August 11, 2015.

20. Healthcare.gov. Who's eligible to use the marketplace. https://www.healthcare.gov/quick-guide/eligibility/. 2015. Accessed August 11, 2015.
21. Michigan Department of Health and Human Services. Michigan HIV/AIDS Drug Assistance Program (MIDAP) Qualifications. http://www.michigan.gov/mdch/0,1607,7-1322940_2955_2982-44913-,00.html. 2015. Accessed August $11,2015$.

22. Health Resources and Services Administration (HRSA). Michigan- Ryan White HIV/AIDS Program Client Characteristics State Profile. 2012. http://hab.hrsa.gov/stateprofiles/ Client-Characteristics.aspx. Accessed February 19, 2015.

23. Michigan Drug Assistance Program. HIV/AIDS Related Treatments: Antiretrovirals. 2015. http://www.scriptguiderx .com/UPLOADS/formularies/032215.pdf. Accessed March $1,2015$.

24. Positively Aware. HIV Drug Guide. 2015. http://www .positivelyaware.com/articles/drugguide. Accessed December 2014.

25. Astuti N, Maggiolo F. Single-tablet regimens in HIV therapy. Infect Dis Ther. 2014;3:1-17.

26. O'Connell DA. The Affordable Care Act: not a cure-all for HIV. Public Administration Review. 2013;73(1):S103S104.

27. Cunningham WE, Hays RD, Duan N, et al. The effect of socioeconomic status on the survival of people receiving care for HIV infection in the United States. J Health Care Poor Underserved. 2005;16:655-676.

28. Kousignian I, Abgrall S, Grabar S, et al. Maintaining antiretroviral therapy reduces the risk of AIDS-defining events in patients with uncontrolled viral replication and profound immunodeficiency. Clin Infect Dis. 2008;46: 296-304.

29. 42 U.S.C. $\S 18031(\mathrm{c})(1)(\mathrm{a})$.

30. Wawrzyniak A, Ownby RL, McCoy K, Waldrop-Valverde D. Health Literacy: Impact on the health of HIV-infected individuals. Curr HIV/AIDS Rep. 2013;10:295-304.

31. Bouffard K. Parents' illiteracy a challenge. 2014. http:// www.detroitnews.com/article/20140131/LIFESTYLE03/ 301310006. Accessed March 16, 2015.

32. Detroit Literacy Coalition. Frequently asked questions. 2008. http://www.detroitliteracy.org/faq.htm. Accessed February $20,2015$.

33. Ntiri D. The silent crisis-illiteracy in Detroit. http://www .highbeam.com/doc/1P1-79470117.html. Accessed March $15,2015$.

34. Berkman ND, Sheridan SL, Donahue KE, Halpern DJ, Crotty K. Low health literacy and health outcomes: an updated systematic review. Ann Intern Med. 2011;155: 97-107.

35. Davis TC, Wolf MS, Bass PF III, et al. Low literacy impairs comprehension of prescription drug warning labels. J Gen Intern Med. 2006;21:847-851.

36. Kalichman SC, Cherry J, Cain D. Nurse-delivered antiretroviral treatment adherence intervention for people with low literacy skills and living with HIV/AIDS. J Assoc Nurses AIDS Care. 2005;16(5):3-15.

Address correspondence to: Ms. Yasamean Zamani-Hank

Department of Health Behavior \& Health Education University of Michigan School of Public Health 1415 Washington Heights Ann Arbor, MI 48109

E-mail: yzamani@umich.edu 